\title{
On the Thermodynamics of a Two-Dimensional Electron Gas with Non-Parabolic Dispersion
}

\author{
G. Gulyamovํㅗ B. T. Abdulazizov² \\ ${ }^{1}$ Pedagogical Institute, Namangan, Uzbekistan \\ ${ }^{2}$ Namangan State University, Namangan, Uzbekistan \\ Email: bt_abdulazizov@mail.ru
}

How to cite this paper: Gulyamov, G. and Abdulazizov, B.T. (2016) On the Thermodynamics of a Two-Dimensional Electron Gas with Non-Parabolic Dispersion. World Journal of Condensed Matter Physics, 6, 294-299.

http://dx.doi.org/10.4236/wjcmp.2016.64028

Received: September 8, 2016

Accepted: November 14, 2016

Published: November 17, 2016

Copyright $\odot 2016$ by authors and Scientific Research Publishing Inc. This work is licensed under the Creative Commons Attribution International

License (CC BY 4.0).

http://creativecommons.org/licenses/by/4.0/

\begin{abstract}
A thermodynamic density of states, electron density in the subband and the entropy of the gas as function of the temperature and the total two-dimensional electron density are studied. Semiconductor conduction band dispersion is described by the simplified Kane model. Numerical simulation shows that with an increase in the total electron concentration, thermodynamic density of states at low temperatures changes abruptly and smoothes jumps at high temperatures. This change manifests itself in the peculiar thermodynamic characteristics. The results are used to interpret existing experimental data.
\end{abstract}

\section{Keywords}

Quantum Well, Two-Dimensional Electron Gas, Kane Model, Subbands Statistics, Entropy

\section{(c) (i) Open Access}

\section{Introduction}

Recently, a large number of studies are devoted to the study of two-dimensional electron gas in a magnetic field [1]-[3]. Studying influences of other factors as anisotropy of the electron spectrum and conduction band nonparabolicity on the properties of two dimensional electron gas is also important [4]. The results of such studies play an important role for the understanding of the nature of the two-dimensional electron gas: subbands structure, electron statistics in subbands etc.

In work [5], it analyzes density of states (DOS) of two-dimensional electron gas in a single quantum well (QW) on the base of narrow-gap semiconductors, InAs and InSb. It is shown that the nonparabolicity of conduction band leads to a noticeable change in the DOS of two-dimensional electron gas.

It is known [6] that most of the experimentally observed thermodynamic properties 
of the electron gas (such as entropy, heat capacity, etc.) are directly determined by the DOS system. Thus, the observed properties of two-dimensional electron gas in a narrow-gap material are primarily determined by the quantization of electron energy and nonparabolicity of conduction band.

This work is devoted to the calculation of the thermodynamic DOS, the concentration of electrons in the subband and the entropy of a gas as a function of total concentration and temperature with allowance for the nonparabolicity of conduction band. It is shown that with increasing total concentration of electrons, thermodynamic DOS changes abruptly, and this leads to a peculiar change in the subband's concentration and entropy. The results are compared with experimental data on the basis of the electron gas in the quantum well heterostructures, InAs/AlSb.

\section{Basic Equations}

Consider a QW width L, concluded between the barriers of infinite height.

The energy is measured from the bottom of the bulk semiconductor. Dispersion law of electrons in the conduction band relies on nonparabolicity, and in the simplest case describe by two-band Kane model. In the effective mass approximation, the solution of the Schrödinger equation leads to the following dispersion

$$
E(1+\alpha E)=\frac{\hbar^{2} k^{2}}{2 m(0)}+\varepsilon_{1} n^{2},
$$

where $k^{2}=k_{x}^{2}+k_{y}^{2}, \quad \varepsilon_{1}=\hbar^{2} \pi^{2} / 2 m(0) L^{2}, m(0)$-electron effective mass at the bottom of the conduction band (in a unit $m_{0}$ ), $\alpha$-parameter nonparabolicity of conduction band.

The total concentration and the concentration of electrons in the subbands defined by the relations

$$
n_{s}=\sum_{n=1} n_{s}^{(n)}, n_{s}^{(n)}=N_{0} \int_{\bar{E}}^{\infty}(1+2 \alpha E) f(E) \mathrm{d} E,
$$

where $N_{0}=m(0) / \pi \hbar^{2}, n_{s}^{(n)}$-concentration $n$-th subband, $\bar{E}_{n}$-solution to Equation (1) in case $k=0, f(E)=(\exp ((E-\mu) / T)+1)^{-1}$-the Fermi-Dirac distribution function, $\mu$ is a chemical potential of electron gas. Equation (2) determine the concentration of electrons in individual subbands $n_{s}^{(i)}\left(n_{s}\right)$ depending on the temperature $T$ and the total concentration $n_{s}$.

The thermodynamic DOS is defined as $g_{s}=\partial n_{s} / \partial \mu$. Given that the $\partial f / \partial \mu=-\partial f / \partial E$, integrating by parts we obtain the following expression

$$
g_{s}=N_{0} \sum_{n=1}\left\{\left(1+2 \alpha \bar{E}_{n}\right) f\left(\bar{E}_{n}\right)-2 \alpha\left(\bar{E}_{n}-\mu\right)+2 \alpha T \ln \left(\mathrm{e}^{\frac{\bar{E}_{n}-\mu}{T}}+1\right)\right\} \text {. }
$$

The thermodynamic potential $\Omega_{S}(\mu, T)$ and entropy $S$ can be found using the following relations

$$
\Omega_{S}(\mu, T)=-T N_{0} \sum_{n=1}^{\infty} \int_{E_{n}}^{\infty}(1+2 \alpha E) \ln \left(1+\mathrm{e}^{\frac{\mu-E}{T}}\right) \mathrm{d} E
$$




$$
S=-\left(\frac{\partial \Omega_{S}}{\partial T}\right)_{\mu}
$$

\section{Numerical Results and Discussions}

With the help of the relations (2)-(5) can provide useful information about the behavior of the two-dimensional electron gas at varying temperatures and the total electron concentration. Calculations of the density of states and entropy are carried out by the example of InAs semiconductor. Used in the calculation of the band parameters of InAs semiconductor are shown in Table 1.

Since the total concentration $n_{s}$, DOS $g_{s}$, and entropy $S$ in the Equations ((2), (3) and (5)) depends on the chemical potential $\mu$ parametrically, we can just build them, depending on the concentration of $g_{s}\left(n_{s}\right)$, of $S\left(n_{s}\right)$. For fixed temperature $T$ these dependences can be obtained from Equations ((2), (3), (5)) by changing the chemical potential $\mu$ in the range of $\sim 0 \div 0.4 \mathrm{eV}$.

Figure 1 shows the dependence of the DOS $g_{s}$ on the total two-dimensional electron concentration $n_{s}$ for QW with width $L=18 \mathrm{~nm}$ and different values of temperature $T$ and the parameter nonparabolicity $\alpha$.

The graph shows the temperature significantly influences the shape of $g_{s}\left(n_{s}\right)$ dependence. The shape of function $g_{s}\left(n_{s}\right)$ is mainly determined by the Fermi-Dirac distribution function at level $\bar{E}_{n}$ (see (3) at $\alpha=0$ ). For zero temperature $f\left(\bar{E}_{n}\right)=0$ if

Table 1. Band parameters of InAs.

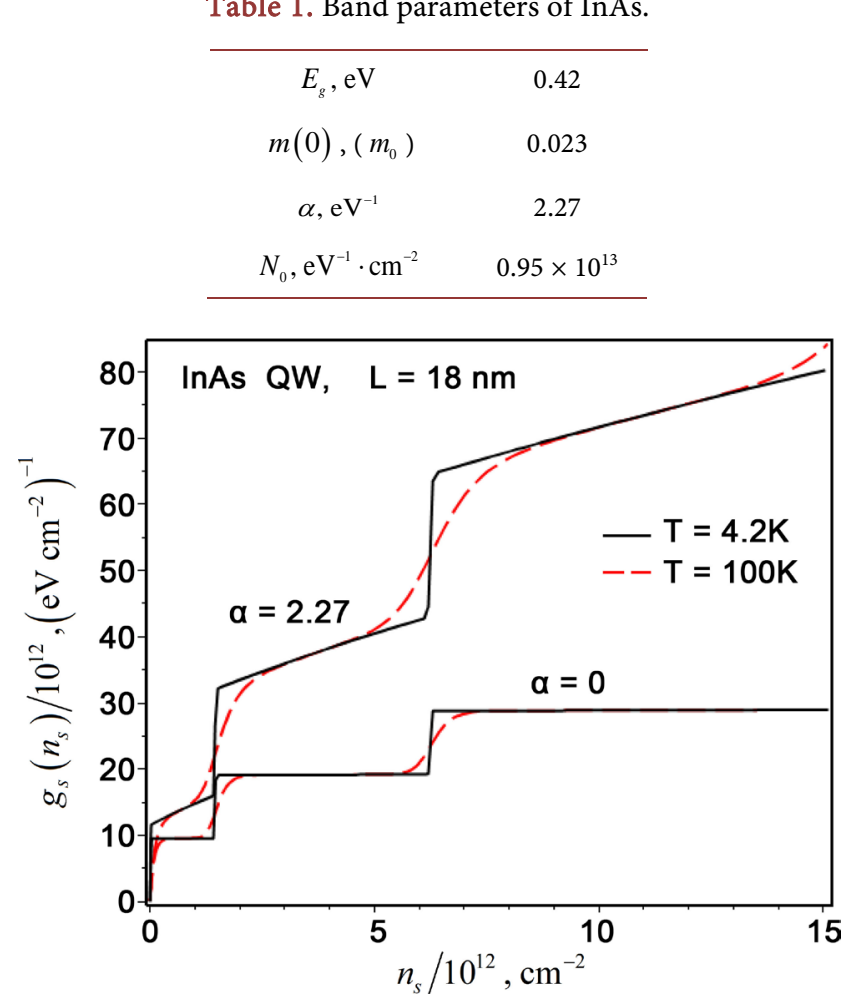

Figure 1. DOS of electron gas as function of the total two-dimensional electron concentration in the InAs QW: $\mathrm{L}=18 \mathrm{~nm}, \mathrm{~T}=4.2$, and $100 \mathrm{~K}, \alpha=0,2.27$. 
$\mu<\bar{E}_{n}$ and $f\left(\bar{E}_{n}\right)=1$ if $\mu>\bar{E}_{n}$. For finite temperature $\mu$ dependence of $f\left(\bar{E}_{n}\right)$ is smoothly. At low temperatures, the broadening of the energy levels of the weak and the DOS has sharply stepped shape. Low temperature has small effect on the shape DOS. The increase in temperature leads to a strong smoothing of the thermodynamic DOS. It significantly changes its shape, a step change in the DOS greatly suppressed, instead of the step appears gradually growing smooth curve. Accounting nonparabolicity electron band leads to the fact that at low temperatures within each subband DOS increases approximately linearly as compared to nonparabolic approach. The jump is also growing.

Since the thermodynamic quantities-entropy, heat capacity, etc. is directly linked to the DOS, and then these values are also abrupt change.

Figure 2 shows the variation of the electron gas entropy as function of the total twodimensional electron concentration at $T=70 \mathrm{~K}, L=18 \mathrm{~nm}$, in the InAs QW with (solid line) and without of conduction band nonparabolicity (dotted line).

The graph shows that the calculation of the thermodynamic quantities-nonparabolicity effects are important. For example, at a concentration $n_{s} \approx 5 \times 10^{12} \mathrm{~cm}^{-2}$, entropy $S / k_{B}$ more than two time large compared to $\alpha=0$.

Nature jumps in the concentration dependence of $g_{s}\left(n_{s}\right), S\left(n_{s}\right)$ is known. They are caused by the overlap of the different subbands. With increasing concentration, the Fermi level crosses the bottom of the next subband DOS dramatically increased [5].

The jump in the concentration dependence $g_{s}\left(n_{s}\right)$ is manifested in a special way as filling of subbands. Figure 3 shows the filling of the subbands with indices $i=1,2,3$, depending on the total two-dimensional electron concentration $n_{s}$ at $T=4.2 \mathrm{~K}$ in the InAs QW, $L=15$ and $18 \mathrm{~nm}$ calculated from Equation (2). Symbols represented to concentration of electrons in the 1-th, 2-th and 3-th subbands (squares, crosses, circles), determined from the Fourier analysis of the Shubnikov-de Haas experiment [7]. Experimental width of the QW (InAs) is $L_{\text {exp }}=15 \mathrm{~nm}$.

With the growth of the total concentration of the first miniband is filled first, the

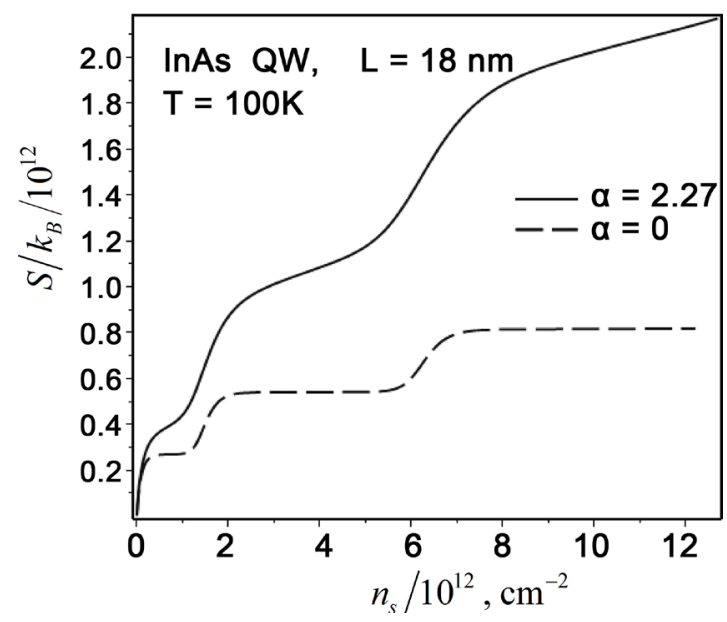

Figure 2. The change of electron gas entropy as function of the total concentration in the InAs QW: $L=18 \mathrm{~nm}, T=100 \mathrm{~K}, \alpha=0,2.27$. 


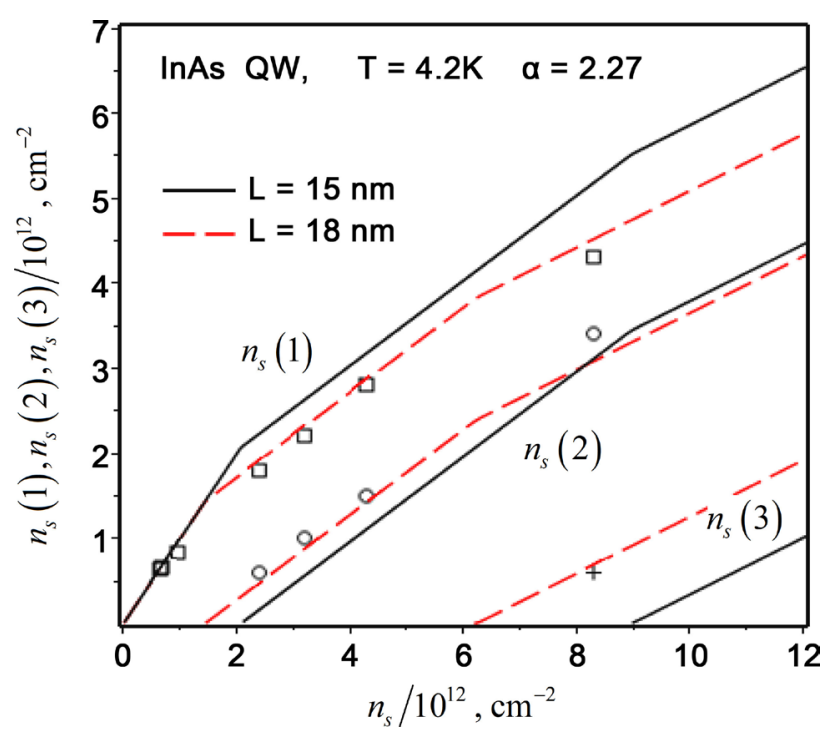

Figure 3. Filling of $i=1,2,3$ subbands, depending on the total two-dimensional electron concentration for InAs QW: $T=4.2 \mathrm{~K}, L=15$ and $18 \mathrm{~nm}$. Symbols-experimental data [7].

concentration continues to increase linearly until it begins filling the second miniband. When filling in the upper subband starts, a filling of the lower subband is slowing. In graphics appears a fracture. These fractures are caused by an abrupt increase in the DOS.

From Figure 3 it appears that the computed curve with $L=18 \mathrm{~nm}$ better coincides with the experimental data compared with $L=15 \mathrm{~nm}$ curve. In fact, used in this study model QW with $V=\infty$ overestimates the energy of the electron.

Our estimates show that if use a real value for the height of the potential barrier (say $V \sim 1 \mathrm{eV}$ ) the calculated curve with $L=15 \mathrm{~nm}$ coincides better with the experimental data for comparison with $L=18 \mathrm{~nm}$ curve.

High temperatures and a wide QW leads to intensive filling of overlying subbands. Figure 4 shows the change in the electron density of the first five subband depending on the total two-dimensional concentration at $T=300 \mathrm{~K}$, in InAs QW, $L=22 \mathrm{~nm}$. In this case, the fractures observed at low temperatures (see Figure 3) are blurred due to the thermal broadening of quantum levels.

\section{Conclusions}

This paper, by using numerical simulation, studied the concentration of electrons in the subbands and the entropy of two-dimensional electron gas, depending on the temperature and the total two-dimensional electron density. To account for the conduction band nonparabolicity in the spectrum (1), a simple Kane model is used.

We have shown that an abrupt change in the DOS with increasing concentration (Figure 1) is shown in the example of the thermodynamic characteristics of the entropy (Figure 2). It was established that in the calculation of the thermodynamic quantities, the role of band nonparabolicity is important.

We also presented numerical results dependences of subbands concentration as 


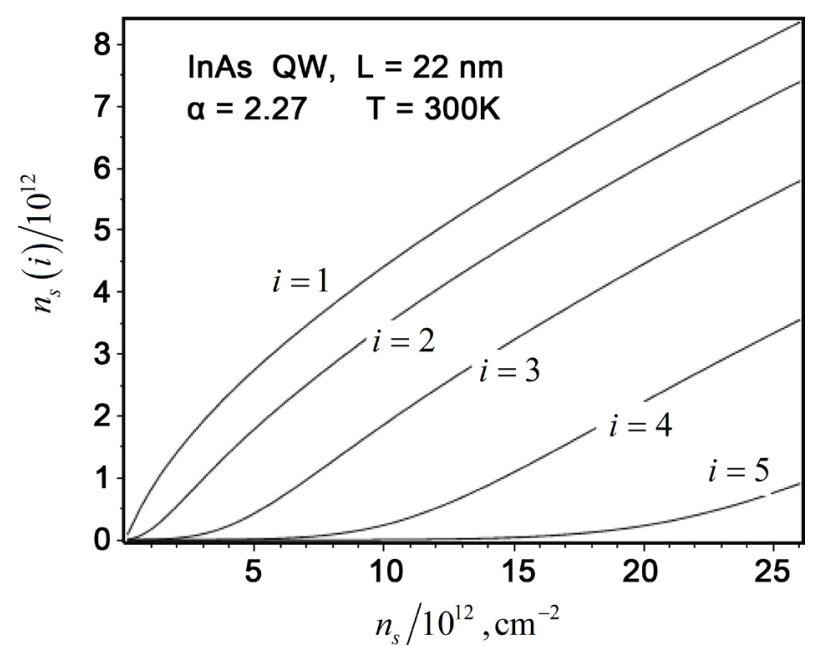

Figure 4. Filling of $i=1,2,3,4,5$ subbands in the InAs QW, depending on the total two-dimensional electron concentration: $T=300 \mathrm{~K}, L=22 \mathrm{~nm}$.

function of total two-dimensional electron concentration (Figure 3). Stepped changes of the DOS are manifested in the form of fracture. These fractures can be seen clearly in calculated lines (Figure 3) and these lines explain the experimental data [7]. The number of filled subbands increases at the wide QW and high temperatures (Figure 4).

\section{Funding}

This work was supported by the Scientific and Technical program Republic of Uzbekistan (Grant F2-OT-O-15494).

\section{References}

[1] Zawadzki, W. (1984) Thermodynamics of Two-Dimensional Electron Gas in a Magnetic Field. Springer Series in Solid-State Sciences. 53, 79-85. http://dx.doi.org/10.1007/978-3-642-82311-4_9

[2] Vagner, D. (2006) Thermodynamics of Two-Dimensional Electron on Landau Levels. HIT Journal of Science and Engineering A, 3, 102-152.

[3] Ramos, A.C.A., Farias, G.A. and Almeida, N.S. (2011) Thermodynamics of a Quasi-two Dimensional Electron Gas: Effects of Magnetic Fields, Temperature and Finite Width. Physic E: Low-Dimensional Systems and Nanostructures, 43, 1878-1881.

[4] Paitya, N. and Ghatak, K.P. (2013) Quantization and Carrier Mass. Reviews in Theoretical Science, 1, 1-141. http://dx.doi.org/10.1166/rits.2013.1008

[5] Gulyamov, G., Baymatov, P.J. and Abdulazizov, B.T. (2016) Effect of Temperature and Band Nonparabolicity on Density of States of Two Dimensional Electron Gas. Journal of Applied Mathematics and Physics, 4, 272-278. http://www.scirp.org/journal/jamp

[6] Askerov, B.M. (1994) Electron Transport Phenomena in Semiconductors. World Scientific, Singapore, 412 p. http://dx.doi.org/10.1142/1926

[7] Aleshkin, V.Y., Gavrilenko, V.I., Ikonnikov, A.V., Sadofyev, Y.G., Bird, J.P., Jonhson, S.R. and Zhang, Y.-H. (2005) Cyclotron Resonance in Doped and Undoped InAs/AlSb Heterostructures with Quantum Wells. Semiconductors, 39, 62-66.

http://dx.doi.org/10.1134/1.1852647 
Submit or recommend next manuscript to SCIRP and we will provide best service for you:

Accepting pre-submission inquiries through Email, Facebook, LinkedIn, Twitter, etc. A wide selection of journals (inclusive of 9 subjects, more than 200 journals)

Providing 24-hour high-quality service

User-friendly online submission system

Fair and swift peer-review system

Efficient typesetting and proofreading procedure

Display of the result of downloads and visits, as well as the number of cited articles

Maximum dissemination of your research work

Submit your manuscript at: http://papersubmission.scirp.org/

Or contact wjcmp@scirp.org 